\title{
The immunoglobulin response to individual HSV-1 viral polypeptides: kinetics of the response during primary and secondary experimental infection with herpes simplex virus.
}

\author{
R. R. MCKENDALL, M. PETTIT and W. WOO
}

Departments of Neurology and Microbiology, The University of Texas Medical Branch, Galveston, Texas 77550, USA

\begin{abstract}
Summary. We studied the immunoglobulin response to individual viral polypeptides in experimental primary and secondary infection with herpes simplex virus (HSV)-1 in mice. After a single footpad inoculation with $10^{5 \cdot 6} \mathrm{pfu}$ of $\mathrm{HSV}-1$, immunoglobulin to proteins mol. wts $\left(10^{3}\right) 44$ and 75 appeared on day 7 . Antibodies to $\mathrm{gB}, \mathrm{gC}, \mathrm{gD}$, $42 \times 10^{3}$ - and $(48-52) \times 10^{3}$-mol. wt proteins appeared on day 11 and antibody to the major capsid protein, VP154, appeared on day 15 after infection. The secondary immune response was characterised by early production of antibody to gD on day 3 followed by antibodies against the $42 \times 10^{3}$ - and $44 \times 10^{3}$-mol. wt proteins on days 4 and 5 respectively. Antibodies to glycoprotein $\mathrm{gC}$ and $\mathrm{gB}$ were delayed until day 7 of the secondary immune response. In both primary and secondary immune responses the responses against proteins of mol. wts $\left(10^{3}\right) 42$ and 44 were particularly intense and of high titre. We conclude that the kinetics of anti-polypeptide antibody appearance is markedly asynchronous; and that the anti-glycoprotein responses occur too late in primary infection to contribute to viral clearance.
\end{abstract}

\section{Introduction}

The serum antibody response in experimental herpes simplex virus (HSV)-1 models appears as early as day 3 or as late as day 12 in various reported studies (Knotts et al., 1974; Rosenberg and Notkins, 1974; Oakes, 1975; Zaia et al., 1975; Rager-Zisman and Allison, 1976; Morahan et al., 1977, 1981). In our previous work with the footpad route of infection, footpad virus titres begin to decrease substantially 7 days after inoculation with virus (McKendall, 1985). Serum antibody activity is detectable 7-10 days after infection by neutralisation assay (McKendall and Baringer, 1981) and 12 days earlier by ELISA or radioimmunoassay. Because the serum antibody titres would be expected to lag behind the levels of antibody produced locally in tissues, it is possible that the earliest antibodies produced during natural infection might contribute to the decline in viral titres. Furthermore, different HSV-1 polypeptides differ in their immunogenicity and in the amount of protein synthesised and presented to the immune system during infection. Therefore, the kinetics of the

Received 20 Oct. 1986; revised version accepted 6 Apr. 1987. antibody response to the individual polypeptides would be expected to be incoordinate. It would be of interest to know which viral polypeptides were the targets of the early antibody response.

Among the many viral peptides known, the glycoproteins (gPs) are of interest because they appear to be biologically important targets of antibody and immune responses. Monoclonal antibodies to gPs are reported to be protective in vivo (Dix et al., 1981; Balachandran et al., 1982; Rector et al., 1982; Kümel et al., 1985). Immunisation with purified gPs or synthetic peptides of gPs is protective (Berman et al., 1985; Dix and Mills, 1985; Eisenberg et al., 1985). Thus, if anti-gP antibody were produced sufficiently early in infection, an important biological antiviral role would be plausible.

We were interested, therefore, to define the kinetics of the antibody response to the individual HSV-1 polypeptides and to identify the protein targets of antibody produced early in infection, particularly around day 7 after infection when viral titres in footpad begin to decline markedly. To accomplish this we employed a sensitive immunoblotting procedure which allows detection of antibody to as many as $33 \mathrm{HSV}-1$ polypeptides including 
many proteins besides the glycoproteins (McKendall et al., 1987).

\section{Materials and methods}

\section{Virus}

HSV-1 (Heitzman) was originally isolated from a patient with pharyngitis. The virus isolate was typed as HSV-1 by neutralisation kinetics, and by sodium dodecyl sulphate-polyacrylamide gel electrophoresis (SDSPAGE) polypeptide pattern with isotopically labelled proteins.

\section{Mice and immunisation procedure}

BALB/c mice (Simonsen, Gilroy, CA, USA) 1-5month old males, were used in all experiments. For the primary immune response, mice were given $4 \times 10^{5} \mathrm{pfu}$ of HSV-1 in the footpad and serum was pooled from groups of three mice bled retro-orbitally at intervals up to day 22 after infection. To study secondary immune responses, an adoptive transfer model was used. Donor mice were immunised with $2 \times 10^{3}$ pfu of HSV-1 by footpad followed by intraperitoneal (ip) doses of $2 \times$ $10^{5} \mathrm{pfu}, 1 \times 10^{6} \mathrm{pfu}$ and $5 \times 10^{7} \mathrm{pfu}$ at $2-4$ week intervals. One month after the last dose, donor spleens were harvested and prepared as a single cell suspension. After washing three times in cold PBS, a viable cell count in fresh trypan blue $(0 \cdot 16 \% \mathrm{w} / \mathrm{v}$ in saline) was performed. Four-month-old recipient mice were given $5.5 \times 10^{7}$ viable immune spleen cells ip; controls received $5.5 \times 10^{7}$ non-immune spleen cells from age- and sex-matched donors. One day later recipients were given $4 \times 10^{5} \mathrm{pfu}$ of HSV-1 in the footpad. Serum was pooled from groups of three mice which were bled at intervals up to day 7 .

\section{Preparation of infected cell-lysate proteins}

Lysates were prepared according to methods described in the preceding paper (McKendall et al., 1987). Briefly, human diploid lung fibroblasts, MRC-5, were grown to confluency and infected with HSV-1 at a multiplicity of $10 \mathrm{pfu} / \mathrm{cell}$. Cells were harvested when the CPE was $4+$, usually after $24 \mathrm{~h}$. The infected cells were suspended in lysate buffer containing SDS $0 \cdot 1 \%$ w/v, Triton-X-100 1\% $\mathrm{v} / \mathrm{v}$ and deoxycholate $1 \% \mathrm{w} / \mathrm{v}, p \mathrm{H} 6.8,0.5 \mathrm{M} \mathrm{NaCl}$, and frozen at $-70^{\circ} \mathrm{C}$ until used. Lysates contained $4.5 \mathrm{mg}$ total protein $/ \mathrm{ml}$.

\section{Sodium dodecyl sulphate-polyacrylamide gel electrophoresis}

SDS-PAGE was performed according to the standard procedure of Laemmli (1970). The details of sample preparation and protein concentration have been described (McKendall et al., 1987). Briefly, an $8.5 \% \mathrm{w} / \mathrm{v}$ acrylamide resolving gel cross-linked with bisacrylamide or DATD and a stacking gel of $3.5 \% \mathrm{w} / \mathrm{v}$ bisacrylamide were employed. An important detail of the procedure is that HSV-1-infected cell-lysate proteins for these studies were loaded at a concentration of $11 \mu \mathrm{g} /$ well in wells $4 \mathrm{~mm}$ wide $\times 0.75 \mathrm{~mm}$ thick. Electrophoresis was performed at $15 \mathrm{~mA}$ constant current on gels $0.75 \mathrm{~mm}$ thick by $14 \mathrm{~cm}$ long and gels were electroblotted.

\section{Western blotting of HSV-1-infected cell polypeptides}

The conditions for optimal transfer of HSV-1-infected cell-lysate polypeptides by Western blotting have been previously described (McKendall et al., 1987). Transfer of proteins to the nitrocellulose paper was accomplished electrophoretically by use of Tris-glycine buffer with methanol $20 \% \mathrm{v} / \mathrm{v}$ as described by Towbin et al., (1979). Transfer was done with a Transphor apparatus (Hoefer Co, San Francisco, CA, USA) at 25 V, 0.2 A overnight.

\section{Detection of antibody to $H S V-1$ polypeptides by immunoblotting}

The conditions and sensitivity of our immunoblotting procedure have been described in the preceding paper (McKendall et al., 1987). The procedure includes a blocking step with bovine serum albumin (BSA) $3 \% \mathrm{w} / \mathrm{v}$, renaturation with Triton-X-100 $0.5 \% \mathrm{v} / \mathrm{v}$, and treatment with a second antibody consisting of affinity purified ${ }^{125} \mathrm{I}$ labelled sheep anti-mouse Ig (Amersham, Arlington Heights, USA). Bands for experiments reported in this paper were detected by autoradiography with Kodak XAR film and exposures ranging from one to 12 days.

\section{Results}

Kinetics and composition of antiviral immunoglobulin response during primary infection

Mice were inoculated with $4 \times 10^{5}$ pfu of HSV-1 in the footpad. Pooled sera from cohorts of infected mice were harvested at intervals after virus inoculation. Before virus inoculation, mice had been bled and the pooled sera shown to be without HSV-1 neutralising activity. The collected sera were subjected to immunoblotting and fig. 1 shows the pattern of polypeptide reactivity which they contained. On days $1-5$, several background bands were found which are non-specifically present in non-immune serum from mice and other species, including man and rabbits (McKendall, unpublished observation). On day 7 , the earliest virusspecific immunoglobulin responses were found. These were directed against proteins of mol. wts $\left(10^{3}\right) 75$ and 44 . Reactivity to both proteins intensified later. On day 11 several additional bands of reactivity appeared including antibody to $\mathrm{gC}$ and $\mathrm{gB}$ in the mol. wt $\left(10^{3}\right)$ range $120-130$, intense reactivity to a protein of mol. wt $42 \times 10^{3}$ and weak reactivity to $\mathrm{gD}$ and to an unidentified protein in 


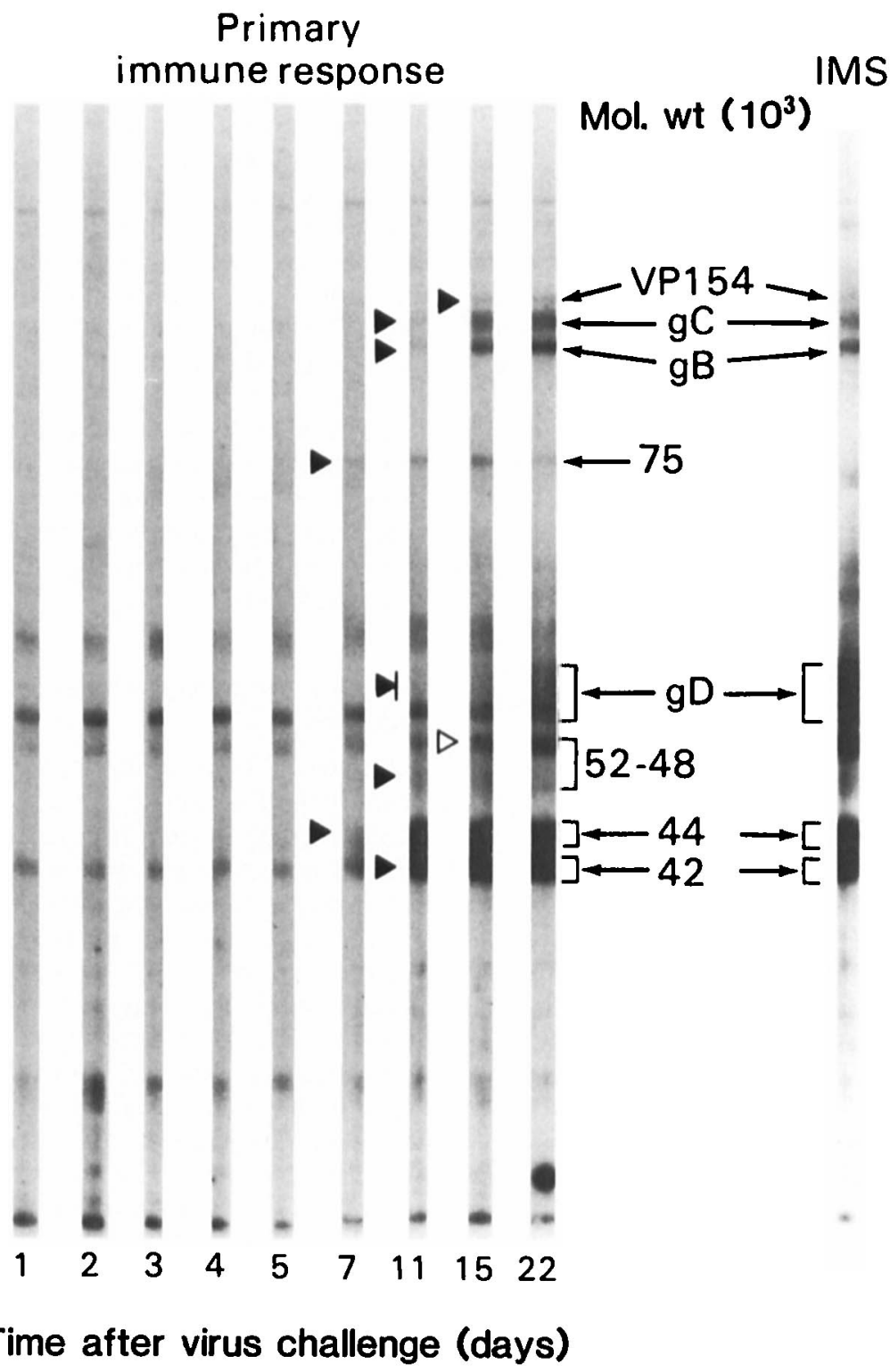

Fig. 1. HSV-1 polypeptides recognised by serum taken from mice at intervals after a primary immunising dose of $4 \times 10^{5}$ pfu of HSV-1. Strips of Western blots containing HSV-1 polypeptides separated by SDS-PAGE were probed with mouse serum taken from day 1 to day 22 after virus challenge. After washing, strips were then probed with ${ }^{125} \mathrm{I}$-labelled sheep anti-mouse Ig. The autoradiograph strips are labelled according to the day serum was harvested. For comparison, a positive control immune mouse serum (IMS) is provided. Solid arrowheads indicate the earliest day on which antibody reactivity was detected for each viral polypeptide. See text for explanation of other symbols.

the mol. wt $\left(10^{3}\right)$ range $48-52$. On day 15 antibody to the last of the major proteins appeared, antibody to the capsid protein of mol. wt $154 \times 10^{3}$. On day 22 , and perhaps on day 15 , there was a band with an apparent mol. wt of c. $52 \times 10^{3}$ (fig. 1, open arrow head). Serum taken earlier showed much fainter reactivity to this band which was considered to be a background band, possibly representing antibody to vimentin, a cell cytoskeletal protein, whose published mol. wt is $52 \times 10^{3}$. Thus the earliest protein targets of immunoglobulin were not the major glycoproteins, and some of the most intense antibody reactivity was noted against proteins of low mol. wt $\left(44 \times 10^{3}\right.$ and $\left.42 \times 10^{3}\right)$. It 
should also be noted that there is no antibody to gE which appears not to be a major immunogenic glycoprotein during primary infection.

\section{Titre of antibody to individual polypeptides in the primary immune response}

We next studied the convalescent serum from day 22 after virus infection to determine the relative titre of antibody to each individual viral protein. Serum dilutions from 1 in 50 to 1 in 100000 were immunoblotted (table) Proteins $\mathrm{gB}, \mathrm{gC}, \mathrm{gD}$, and proteins of mol. wts $\left(10^{3}\right) 44$ and 42 were all detected at serum dilutions up to 1 in 20000 ; these were clearly the most immunogenic proteins based on this criterion. VP154, the capsid protein, was detectable at a dilution of 1 in 1600 and the protein of mol. wt $75 \times 10^{3}$ was seen at a dilution of 1 in 800 . Thus, although antibody to the glycoproteins appeared later than antibody to the proteins of mol. wts $\left(10^{3}\right) 44$ and 75 , equally high titres were ultimately achieved.

\section{Kinetics and composition of antiviral antibody response during secondary infection}

To determine if the antibody responses to the proteins of mol. wts $\left(10^{3}\right) 44$ and 75 would precede anti-gP responses during a secondary immune response, we employed an adoptive transfer model. This was used because circulating antiviral antibody from previous primary infection would mask or neutralise, or both, the challenge dose of virus. Donor mice were immunised with four doses of live HSV-1. Donor spleens were harvested and an injection of whole spleen suspension containing $5 \cdot 5 \times 10^{7}$ splenocytes was given intraperitoneally to histocompatible mice. Non-immune spleen from age- and sex-matched donors which were not immunised was adoptively transferred as a control into histocompatible recipient mice. Mice were then challenged with $4 \times 10^{5}$ pfu of HSV-1 by footpad inoculation and pooled serum from cohorts of both groups of recipient mice was studied by immunoblotting.

Fig. 2 shows the immunoblotting reactivity of the serum from these mice. The polypeptide specificity of the antibody that appeared earliest was that of anti-gD immunoglobulin detected on day 3 after virus challenge. Production of anti-gD antibody intensified strongly on days 4,5 and 7 . The next antibodies detected were against the proteins of mol. wts $\left(10^{3}\right) 44$ and 42 . These appeared on day 4 and intensified but more slowly than anti$\mathrm{gD}$ antibody. Finally of antibodies to the major viral proteins, anti-gB/gC appeared very faintly on day 5 and more intensely on the 7 th day. In addition antibodies to some minor low-mol. wt proteins were observed on day 7 (open arrows) as well as a diffuse area of faint reactivity between $\mathrm{gD}$ and $\mathrm{gB} / \mathrm{gC}$. For comparison, the reactivity pattern of serum from mice immunised with three doses of live virus is provided in lane HIMS and shows fundamentally the same major proteins in addition to some minor ones. Thus the secondary immune response was led by anti-gD significantly before other antibodies appeared and also contained a prominent early response to the proteins of mol. wts $\left(10^{3}\right) 44$ and 42 . The controls which received non-immune spleen showed no HSV-1 specific reactivity until day 7 when an early response to the proteins of mol. wts $\left(10^{3}\right) 44$ and 42 was seen, as in the primary immune response shown in fig. 1 .

\section{Comparison of primary and secondary immune responses}

For visual comparison, the differences between primary and secondary infection have been displayed in fig. 3 according to the time of earliest appearance. Anti-gD was most noticeable in the secondary response, appearing on day 3 whereas it appeared on day 11 in the primary response. Antibody to the $42 \times 10^{3}$-mol. wt protein was found nearly as early. Antibody to $44 \times 10^{3}$-mol. wt protein was least accelerated being only 2 days earlier in the secondary response. However the $44 \times 10^{3}-\mathrm{mol}$. wt protein remained among the earliest targets in the secondary and primary responses. Antibody to the capsid protein, VP154, was not seen in the secondary response up to and including day 7 , but may have appeared later.

\section{Discussion}

The immunoglobulin response to $\mathrm{HSV}-1$ polypeptides has been shown to be strikingly asynchronous in the times of appearance of antibody to the individual proteins. The gPs are not the targets of the early antibody produced during primary infection and the appearance of anti-gB, $-\mathrm{gC}$ and $-\mathrm{gD}$ at day 11 is much too late for these antibodies to have an important role in virus clearance. Even assuming that some anti-gP antibody may be available 1 or 2 days before the appearance of detectable serum titres, anti-gP antibody could not account for any of the footpad virus clearance which occurs 5-7 days after footpad inoculation.

Other studies have also found significant variation in the kinetics of the different anti-polypeptide 

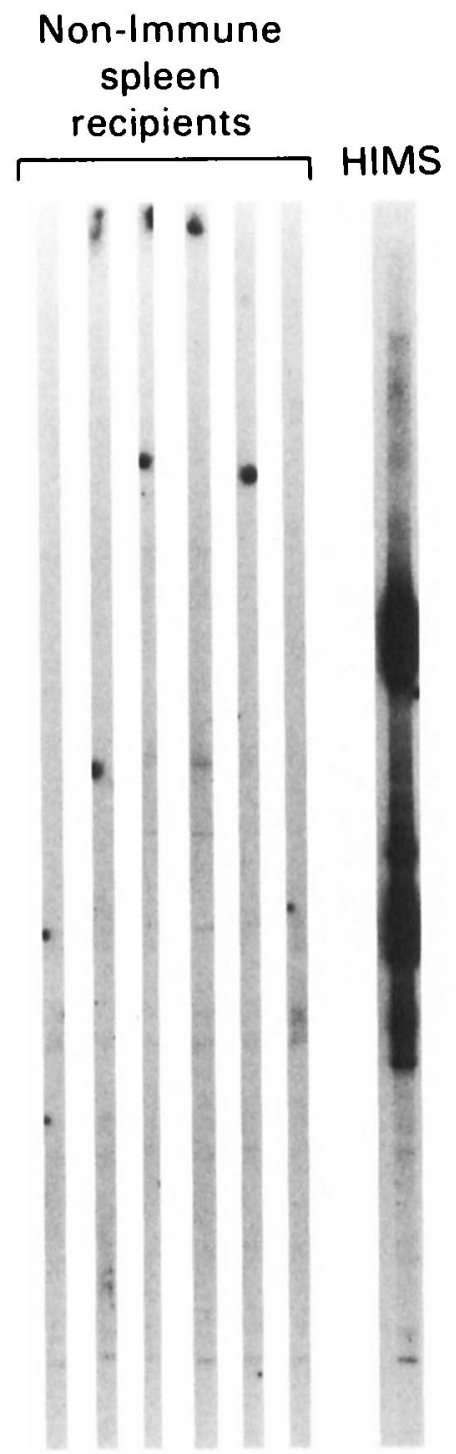

$\begin{array}{llllll}1 & 2 & 3 & 4 & 5 & 7\end{array}$
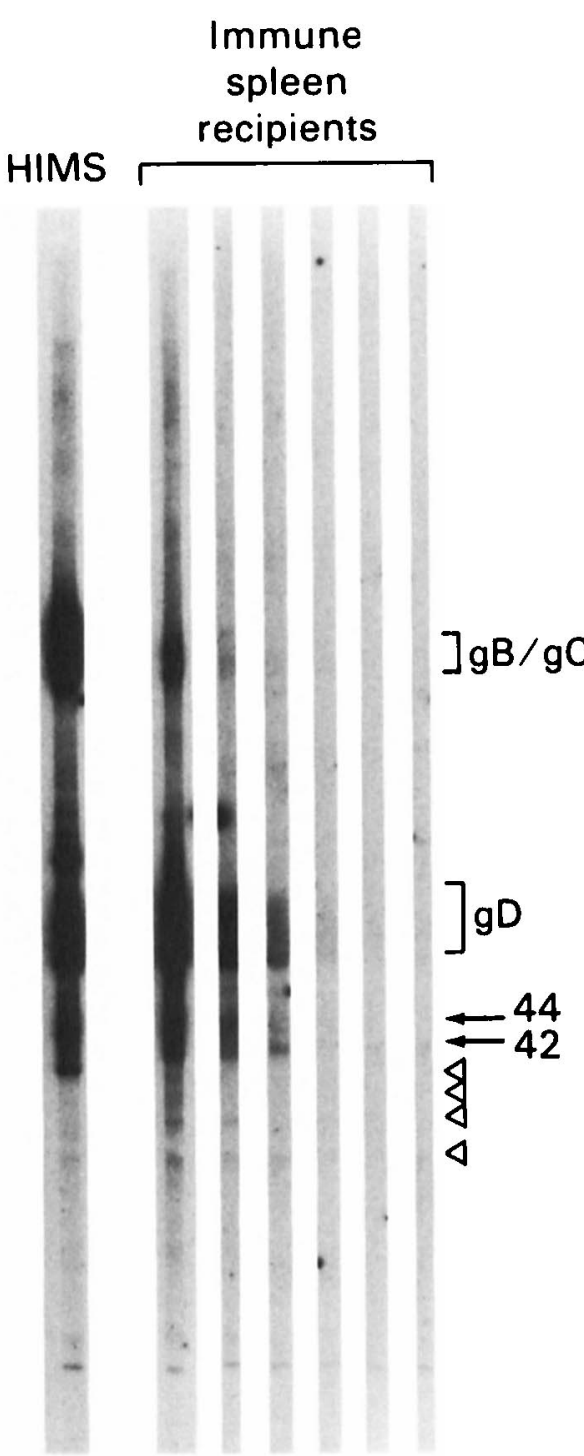

$\begin{array}{llllll}7 & 5 & 4 & 3 & 2 & 1\end{array}$

\section{Time after virus challenge (days)}

Fig. 2. Autoradiographs of immunoblots showing HSV-1 polypeptides recognised by antibody produced during a secondary immune response. Mice were adoptively immunised with immune whole spleen cells and then challenged with $4 \times 10^{5}$ pfu of $\mathrm{HSV}-1$ one day later. Serum was taken on days 1, 2, 3, 4, 5 and 7 after virus challenge. The immunoblot strips are numbered according to the day on which serum was harvested. Control mice were adoptively immunised with non-immune whole spleen cells and then challenged with $4 \times 10^{5} \mathrm{pfu}$ of HSV-1. As a positive control hyperimmune HSV-1 serum was also immunoblotted (HIMS). Major bands of reactivity are designated by letter or apparent mol.wt; minor bands are indicated by open arrowheads.

responses. Mann and Hilty (1982) found antibodies to the $159 \times 10^{3}$ and $152 \times 10^{3}-\mathrm{mol}$. wt proteins on day 9 , to the $125 \times 10^{3}$ and $63 \times 10^{3}$-mol. wt proteins on day 14 and to the $132 \times 10^{3}$-mol. wt protein on day 25 . The later appearance of antibodies in their study than in ours may be related to their use of the immunoprecipitation assay. Studies by Eberle and Mou (1983) and Eberle et al. (1985) found variation between patients in polypeptides recognised by acute and convalescent sera collected during pri- 
Table. Antibody titres to individual HSV-1 viral proteins in convalescent serum from primary infection

\begin{tabular}{lc}
\hline Protein & $\begin{array}{c}\text { Antibody } \\
\text { titre* }\end{array}$ \\
\hline VP154 & 1600 \\
gC & 20000 \\
gB & 20000 \\
$75 \times 10^{3}$ mol.wt & 800 \\
gD & 20000 \\
$44 \times 10^{3}$ mol.wt & 20000 \\
$42 \times 10^{3}$ mol.wt & 20000 \\
\hline$*$ Highest serum dilution that gave a \\
positive result in an immunoblotting assay.
\end{tabular}

mary HSV-1 infection. Kahlon et al. (1986) have also recently emphasised the variability of human antibody responses to individual HSV polypeptides. In the latter two studies, some of the differences between patients may have been due to the asynchronous responses we have observed coupled with different times of collection of sera. In addition, other factors such as virus burden and individual differences in immunoresponsiveness among patients probably contribute to the variation in antipolypeptide responses reported. The basis of the variable and asynchronous responses appears to be complex and has not been thoroughly investigated.

The antibody responses to the proteins of molwts $\left(10^{3}\right) 44$ and 75 as early as day 7 raises the possibility that they could contribute to an antiviral effect. However, there are no data available which determine whether these two proteins are biologically important targets of the immune response. Our laboratory has observed that the $75 \times 10^{3}-\mathrm{mol}$. wt protein is an important protein recognised by hyperimmune mouse serum. The protein is not recognised by non-immune mouse serum and immune mouse serum does not recognise a similar protein in uninfected cell-lysate blots (McKendall et al, 1987). Therefore, the $75 \times 10^{3}$-mol. wt protein appears to be of virus not cell origin. One possibility is that it might be a precursor form of another major immunogenic protein, possibly a non-glycosylated form of $\mathrm{gC}$. If so, the antibody to the $75 \times$ $10^{3}$-mol. wt protein might be biologically active in neutralisation or in antibody-dependent cytolytic processes. However, the identity of this protein has not yet been conclusively determined and, therefore, the importance of the early antibody response is unclear.

The identity of the $44 \times 10^{3}-\mathrm{mol}$. wt protein is more certain. Antibody responses to proteins with mol-wts $\left(10^{3}\right)$ in the range $34-49$ have been previously recognised as a prominent component of human humoral responses by Eberle's group (Eberle and Mou, 1983; Eberle et al., 1984, 1985) and by Bernstein et al. (1984). Eberle et al. (1985) further showed that the $(34-49) \times 10^{3}$-mol. wt proteins were bound by monoclonal antibody ID4, which is known to recognise a nucleocapsid protein of mol. wt $40 \times 10^{3}$ present in infected cells (Zweig et al., 1980; Heilman et al., 1981; Preston et al., 1983). The anti-p40 response has been found to be the earliest anti-polypeptide response in some studies of human sera. Eberle et al. (1985) found

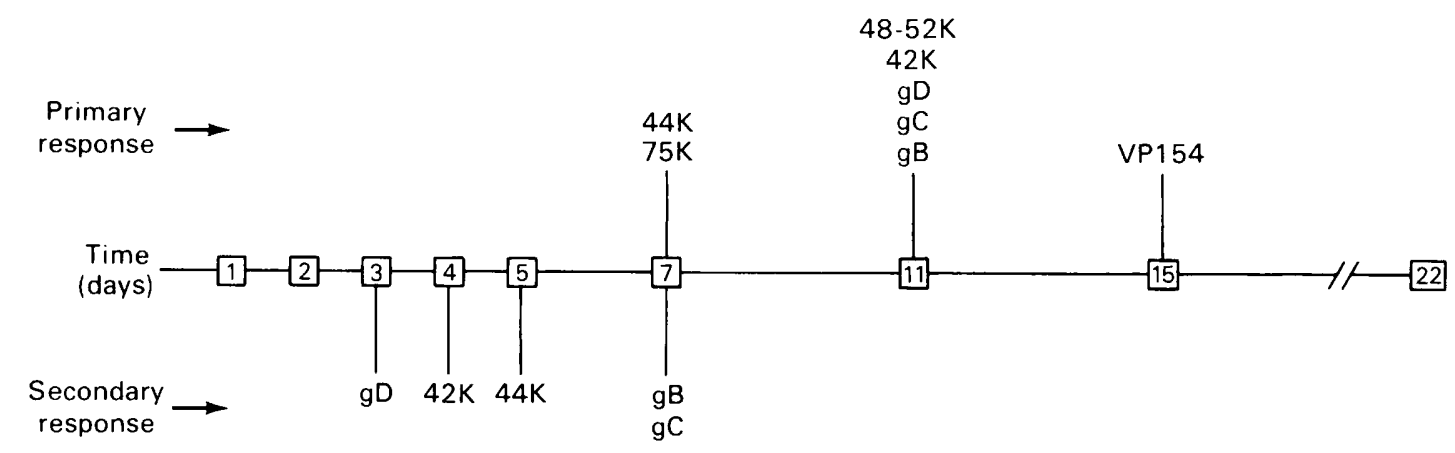

Fig. 3. Day of earliest appearance of antibody to individual HSV-1 proteins, designated by letter or apparent mol. wt $\left(10^{3}\right)$, during primary or secondary immune response. 
anti-p40 antibody in acute phase sera in four of eight patients with primary $\mathrm{HSV}-1$ infection and also in some patients with primary HSV-2 infections (Eberle et al., 1984). In contrast Kahlon et al. (1986) studied five patients with primary HSV-1 infections and found that antibody responses to these lowmol. wt proteins appeared relatively late after infection and tended to correlate with genital sites of infection by either HSV-1 or HSV-2. However, many patients in their study were not bled often enough in the critical period 4-10 days after infection to determine how early the response occurred. Our findings are in agreement with those of Eberle et al. and indicate that the anti-p40 response is an early and important component of the immunoglobulin response in both primary and

\section{REFERENCES}

Balachandran N, Bacchetti S, Rawls W E 1982 Protection against lethal challenge of BALB/c mice by passive transfer of monoclonal antibodies to five glycoproteins of herpes simplex virus type 2. Infection and Immunity 37:1132-1137.

Berman P W, Gregory T, Crase D, Lasky L A 1985 Protection from genital herpes simplex virus type 2 infection by vaccination with cloned type 1 glycoprotein D. Science 227: 1490-1492

Bernstein D I, Lovett M A, Bryson Y J 1984 The effects of acyclovir on antibody response to herpes simplex virus in primary genital herpetic infections. Journal of Infectious Diseases $150: 7-13$.

Dix R D, Mills J 1985 Acute and latent herpes simplex virus neurological disease in mice immunised with purified virusspecific glycoprotein $\mathrm{gB}$ or glycoprotein gD. Journal of Medical Virology 17:9-18.

Dix R D, Pereira L, Baringer J R 1981 Use of monoclonal antibody directed against herpes simplex virus glycoproteins to protect mice against acute virus-induced neurological disease. Infection and Immunity 34: 192-199.

Eberle R, Mou S-W 1983 Relative titers of antibodies to individual polypeptide antigens of herpes simplex virus type 1 in human sera. Journal of Infectious Diseases 148:436444.

Eberle R, Mou S-W, Zaia J A 1984 Polypeptide specificity of the early antibody response following primary and recurrent genital herpes simplex virus type 2 infections. Journal of General Virology 65: 1839-1843.

Eberle R, Mou S-W, Zaia J A 1985 The immune response to herpes simplex virus: Comparison of the specificity and relative titers of serum antibodies directed against viral polypeptides following herpes simplex virus type 1 infections. Journal of Medical Virology 16:147-162.

Eisenberg R J et al. 1985 Synthetic glycoprotein D-related peptides protect mice against herpes simplex virus challenge. Journal of Virology 56:1014-1017.

Heilman C J, Zweig M, Hampar B 1981 Herpes simplex virus type 1 and 2 intracellular p40: type-specific and crossreactive antigenic determinants on peptides generated by partial proteolysis. Journal of Virology 40:508-515.

Kahlon J, Lakeman F D, Ackermann M, Whitley R J 1986 Human antibody response to herpes simplex virus-specific polypeptides after primary and recurrent infection. Journal of Clinical Microbiology 23: 725-730. secondary infection. Because the p40 antigens are nucleocapsid components and would not be present on cell membrane or on virion envelope, these early antibodies could not be directly antiviral. However, an indirect contribution of immune complex activation of complement or interaction of immune complexes with Fc receptor-bearing cells could be involved. Previous observations that non-neutralising monoclonal antibodies, which also lack any capacity for complement-dependent and cell-dependent cytolysis, are protective in vivo supports the notion that antibody may participate in indirect antiviral defence (Rector et al., 1982). What mechanisms are involved and how biologically important they may be remains to be determined in future studies.

Knotts F B, Cook M L, Stevens J G 1974 Pathogenesis of herpetic-encephalitis in mice after ophthalmic inoculation. Journal of Infectious Diseases 130:16-27.

Kümel G, Kaerner H C, Levine M, Schroder C H, Glorioso J C 1985 Passive immune protection by herpes simplex virusspecific monoclonal antibodies and monoclonal antibodyresistant mutants altered in pathogenicity. Journal of Virology 56:930-937.

Laemmli UK 1970 Cleavage of structural proteins during the assembly of the head of bacteriophage T4. Nature 227:680685.

Mann D R, Hilty M D 1982 Antibody response to herpes simplex virus type 1 polypeptides and glycoproteins in primary and recurrent infection. Pediatric Research 16: 176180.

McKendall R R 1985 IgG-mediated viral clearance in experimental infection with herpes simplex virus type 1 : Role for neutralization and Fc-dependent functions but not $C^{\prime}$ Cytolysis and C5 Chemotaxis. Journal of Infectious Diseases $151: 464-470$.

McKendall R R, Baringer J R 1981 Evidence for B-cell effectiveness on peripheral challenge by HSV-1 in mice. In: A. J. Nahmias et al. (eds) The Human Herpesviruses: an interdisciplinary perspective. Elsevier/North Holland Publishers, New York, p 647.

McKendall R R, Pettit M, Woo W 1987 The immunoglobulin response to HSV-1 viral proteins: Determination of immunogenic proteins and relative antibody titers to individual polypeptides by immunoblotting. Journal of Medical Microbiology 24:49-57.

Morahan P S, Breinig M C, McGeorge M B 1977 Immune responses to vaginal or systemic infection of $B A L B / c$ mice with herpes simplex virus type 2. Journal of Immunology 119:2030-2036.

Morahan P S, Thomson T A, Kohl S, Murray B K 1981 Immune responses to labial infection of $\mathrm{BALB} / \mathrm{c}$ mice with herpes simplex virus type 1 . Infection and Immunity 32:180-187.

Oakes J E 1975 Role for cell-mediated immunity in the resistance of mice to subcutaneous herpes simplex virus infection. Infection and Immunity 12:166-172.

Preston V G, Coates J A V, Rixon F J 1983 Identification and characterization of a herpes simplex virus gene product required for encapsidation of virus DNA. Journal of Virology 45: $1056-1064$. 
Rager-Zisman B, Allison A C 1976 Mechanism of immunologic resistance to herpes simplex virus 1 (HSV-1) infection. Journal of Immunology 116:35-40.

Rector J T, Lausch R N, Oakes J E 1982 Use of monoclonal antibodies for analysis of antibody-dependent immunity to ocular herpes simplex virus type 1 infection. Infection and Immunity 38: 168-174.

Rosenberg G L, Notkins A L 1974 Induction of cellular immunity to herpes simplex virus: Relationship to the humoral immune response. Journal of Immunology 112: 1019-1025.
Towbin H, Staehelin T, Gordon J 1979 Electrophoretic transfer of proteins from polyacrylamide gels to nitrocellulose sheets: Procedure and some applications. Proceedings of the National Academy of Sciences of the USA 76:4350-4354.

Zaia J A, Palmer E L, Feorino P M 1975 Humoral and cellular immune responses to an envelope-associated antigen of herpes simplex virus. Journal of Infectious Diseases 132: 660666.

Zweig M, Heilman C J, Rabin H, Hampar B 1980 Shared antigenic determinants between two distinct classes of proteins in cells infected with herpes simplex virus. Journal of Virology 35:644-652. 